\title{
EGU21-9667
}

https://doi.org/10.5194/egusphere-egu21-9667

EGU General Assembly 2021

(c) Author(s) 2021. This work is distributed under

the Creative Commons Attribution 4.0 License.

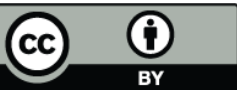

\section{Cosmic-ray muography applications in underground tunnelling}

\author{
Pasi Kuusiniemi ${ }^{1,2}$, Marko Holma ${ }^{1,2,3}$, and Zongxian Zhang ${ }^{4}$ \\ ${ }^{1}$ Muon Solutions Oy, Pyhäsalmi, Finland (pasi.kuusiniemi@muon-solutions.com) \\ ${ }^{2}$ Arctic Planetary Science Institute, Äänekoski, Finland \\ ${ }^{3}$ Kerttu Saalasti Institute, University of Oulu, Nivala, Finland (marko.holma@oulu.fi) \\ ${ }^{4}$ Oulu Mining School, University of Oulu, 90014 Oulu, Finland
}

The novel geophysical remote imaging method of muography is based on cosmic-ray induced muon particles that are detected after passing through the media of interest. If the studied objects are solid, their sizes can vary from meters to up to kilometres. In terms of penetration capability, muography can be placed between methods based on X-rays and those using seismic waves. The most famous objects imaged with muography are pyramids (e.g., Khufu's Pyramid at Giza in Egypt) and volcanoes (e.g., Mt Etna in Italy). One clear advantage of muography compared with seismic methods is that muons, unlike seismic waves, do not reflect from geological interfaces. In addition, the scattering phenomenon is a minor issue and needs consideration only at low-energy muons. Raw data must be corrected according to topography. On the basis of extensive numeric simulations of Hivert et al. (2017), the lowest density variations observable for muography with a significant level of $3 \sigma$ (a typical significance level in physics) are around $2 \%$ at $150 \mathrm{~m}, 4 \%$ at $300 \mathrm{~m}$, and $10 \%$ at $700 \mathrm{~m}$ of depth, respectively. If these numbers are extrapolated to depths below 100 $\mathrm{m}$, the mean density differences in the range of $1 \%$ are likely within the observation capability of muography. It is also worth to note that the $1 \%$ difference in a mean rock density results in an approximately $3 \%$ difference in the muon flux. This indicates that muon flux measurements are very sensitive to the density variations of rocks.

In underground tunnelling, muography has at least four applications: (1) muography can be used to detect a potential risk (such as a water reservoir, a weak zone with loose rocks, boulders, etc.) before or during tunnelling, (2) muography can be employed to monitor overburden rock behaviour during tunnelling operation to avoid risks like the roof cave-ins, (3) muography can be applied to monitor the overburdening rock masses in tunnels after they are excavated to predict and avoid the collapse of rock mass, and (4) muography can be used to estimate the size and volume of a rock mass collapse in a tunnel since the volume of the collapsed rocks must have markedly smaller density than original overburden rock mass. In an excavating tunnel project using a tunnel boring machine (TBM), a muon detector can be installed in the TBM during tunnelling. If there occurs a tunnel cave-in, muography can be employed in undamaged tunnels nearby (sideways or below) the collapse. If possible, the collapse can also be approached safely via an undamaged part of the collapsed tunnel. If none of these are available, borehole muography can be applied as a substitute solution. Whereas an undamaged underground tunnel is either 
filled by air or water, a collapsed tunnel segment is characterized by air and rock, or water and rock. In either case, the average density of the tunnel segment is increased. We are currently planning simulations and real-world tests to validate these assumptions. 